\title{
Temperature Dependence of Isotopic Quantum Effects in Water
}

\author{
R. T. Hart and C. J. Benmore* \\ Intense Pulsed Neutron Source, Argonne National Laboratory, Argonne, Illinois 60439, USA
}

J. Neuefeind

Spallation Neutron Source, Oak Ridge National Laboratory, Oak Ridge, Tennessee 37831, USA

S. Kohara

Japan Synchrotron Radiation Research Institute, 1-1-1 Kouto, Mikazuki-cho, Sayo-gun, Hyogo 679-5198, Japan

\author{
B. Tomberli and P. A. Egelstaff \\ Department of Physics, University of Guelph, Guelph, Ontario, N1G 2W1, Canada
}

(Received 10 September 2004; published 3 February 2005)

\begin{abstract}
The technique of high energy $\mathrm{x}$-ray diffraction has been used to measure the temperature variation of hydrogen versus deuterium isotopic quantum effects on the structure of water. The magnitude of the effect is found to be inversely proportional to the temperature, varying by a factor of 2.5 over the range 6 to $45^{\circ} \mathrm{C}$. In addition, the $\mathrm{H}_{2}{ }^{16} \mathrm{O}$ versus $\mathrm{H}_{2}{ }^{18} \mathrm{O}$ effect has been measured at $26^{\circ} \mathrm{C}$ and the structural difference shown to be restricted to the nearest neighbor molecular interactions. The results are compared to recent simulations and previously measured isochoric temperature differentials; additionally, implications for H/D substitution experiments are considered.
\end{abstract}

DOI: 10.1103/PhysRevLett.94.047801

PACS numbers: 61.20.-p, 61.10.-i, 82.30.Rs

The quantum mechanical nature of the hydrogen bond in water is of fundamental importance and has wide ranging implications for understanding the structure of aqueous solutions and biological processes. The development of new experimental techniques such as isotopic substitution in high energy $\mathrm{x}$-ray diffraction provides a new way of extracting detailed structural information on the quantum mechanics of the hydrogen bond in the liquid state $[1,2]$. These experiments are made possible since electromagnetic radiation experiments are nearly isotope independent (because the radiation scatters from the electron density surrounding the nucleus), enabling the observation of small structural variations between different isotopically enriched samples. For water, the largest isotope effects are manifested in the $\mathrm{O}-\mathrm{O}$ partial radial distribution function, making high energy $\mathrm{x}$-ray diffraction an ideal probe for direct comparison to theory [3]. Moreover, the reduced librational amplitudes and straighter hydrogen bonds of $\mathrm{D}_{2} \mathrm{O}$ compared to $\mathrm{H}_{2} \mathrm{O}$ at the same temperature have been shown to be analogous to a temperature effect, where $\mathrm{D}_{2} \mathrm{O}$ at $23^{\circ} \mathrm{C}$ has a similar structure to that of $\mathrm{H}_{2} \mathrm{O}$ at $17^{\circ} \mathrm{C}$ [1]. Experimental advances have been paralleled in recent years by developments in molecular dynamics using Feynman-Hibbs (FHMD) path-integral and Car-Parrinello (CPMD) approaches [3-7]. Although simulations qualitatively reproduce the measured structural isotopic quantum difference, there is a large variation in the magnitude of different calculations.

Despite their importance in accurately describing the hydrogen bond, little is known about the variation of quantum effects in water with temperature and pressure. An existing FHMD simulation has predicted that there is hardly any change in the magnitude of the difference between the x-ray radial distribution functions of light and heavy water between room temperature and the boiling point [5]. In this Letter, we have carried out $\mathrm{x}$-ray diffraction experiments on $\mathrm{H}_{2} \mathrm{O}$ and $\mathrm{D}_{2} \mathrm{O}$ over the temperature range 6 to $45^{\circ} \mathrm{C}$, which show that the magnitude of the measured isotopic quantum effect is in fact inversely proportional to the temperature, varying by a factor of 2.5 over this temperature range. In addition, experiments on $\mathrm{H}_{2}{ }^{16} \mathrm{O}$ and $\mathrm{H}_{2}{ }^{18} \mathrm{O}$ have been carried out to investigate the role of center-of-mass quantization. The structural isotope difference for the ${ }^{16} \mathrm{O}$ versus ${ }^{18} \mathrm{O}$ substitution is found to be approximately one quarter of the magnitude of the observed $\mathrm{H}$ versus $\mathrm{D}$ effect.

The experimental results presented here provide a rigorous test of intermolecular potentials and $a b$ initio quantum mechanical calculations for water. The largest corrections to the classical model of molecular interactions are believed to be caused by the coupling of intramolecular and intermolecular modes via hydrogen bonding [8], by differences in their ground state librations and by many body effects $[5,9]$. While ab initio calculations, in principle, take these effects into account [7], agreement between the magnitude of the measured and predicted $\mathrm{H}$ versus $\mathrm{D}$ isotope effects, and how they change with temperature, helps to establish the validity in estimating the size of these contributions. These experiments also provide information on the accuracy of the H/D substitution technique in neutron scattering experiments at different temperatures, where the difference in intermolecular structure between isotopically labeled samples is approximated as zero [10]. 
The $\mathrm{H}_{2}{ }^{16} \mathrm{O}$ versus $\mathrm{H}_{2}{ }^{18} \mathrm{O}$ experiments were conducted at the high energy $\mathrm{x}$-ray diffraction beam line, BL04B2 at SPring- 8 with $61.62 \mathrm{keV}$ photons, and the $\mathrm{H}_{2} \mathrm{O}$ versus $\mathrm{D}_{2} \mathrm{O}$ temperature dependence measurements were performed at the BW5 beam line, DORIS III at DESY (HASYLAB), using $110 \mathrm{keV}$ photons. Samples were obtained from Isotec (Miamisburg, OH, SPring-8) and CIL (Cambridge, MA, DESY) at the following enrichments: $\mathrm{H}_{2}{ }^{16} \mathrm{O}$ (99.99\%), $\mathrm{H}_{2}{ }^{18} \mathrm{O}\left(1.7 \%{ }^{17} \mathrm{O}, 2.7 \%{ }^{16} \mathrm{O}\right), \mathrm{D}_{2} \mathrm{O}(99.96 \%)$, $\mathrm{H}_{2} \mathrm{O}$ (2-3 ppm D). The experiments were performed as described in Tomberli et al. [11]. For the temperature dependant studies the $\mathrm{H}_{2} \mathrm{O}$ and $\mathrm{D}_{2} \mathrm{O}$ samples contained in thin walled $(10 \mu \mathrm{m})$ silica tubes were mounted side by side in the same copper block, and the beam variation and other time dependent effects were reduced by interleaving several scans on each isotope. The diffraction data were corrected for incident beam polarization, detector dead time, and container and background scattering as described in Ref. [1]. The electronic structure factors, $S_{X}(Q)$, were obtained by normalizing the high- $Q$ data (between $Q=10$ to $18 \AA^{-1}$, DESY and $Q=7.5$ to $25 \AA^{-1}$, SPring-8) to the isotropic form factor plus Compton scattering [12].

The difference in electronic structure factors between two isotopic water samples can be written as

$$
\begin{aligned}
\Delta S_{X}^{\text {light-heavy }}(Q) & =I^{\text {light }}(Q)-I^{\text {heavy }}(Q) \\
& =\Delta D(Q)+\Delta\left\langle F^{2}\right\rangle+\Delta C(Q),
\end{aligned}
$$

where $I(Q)$ is the fully corrected and normalized measured X-ray data. Light water refers to $\mathrm{H}_{2} \mathrm{O}$ or $\mathrm{H}_{2}{ }^{16} \mathrm{O}$ and heavy water to $\mathrm{D}_{2} \mathrm{O}$ or $\mathrm{H}_{2}{ }^{18} \mathrm{O}$, respectively. $\Delta D(Q)$ is the difference in the intermolecular structure factors, $\Delta\left\langle F^{2}\right\rangle$ is the difference intramolecular scattering [1], and $\Delta C(Q)$ is the

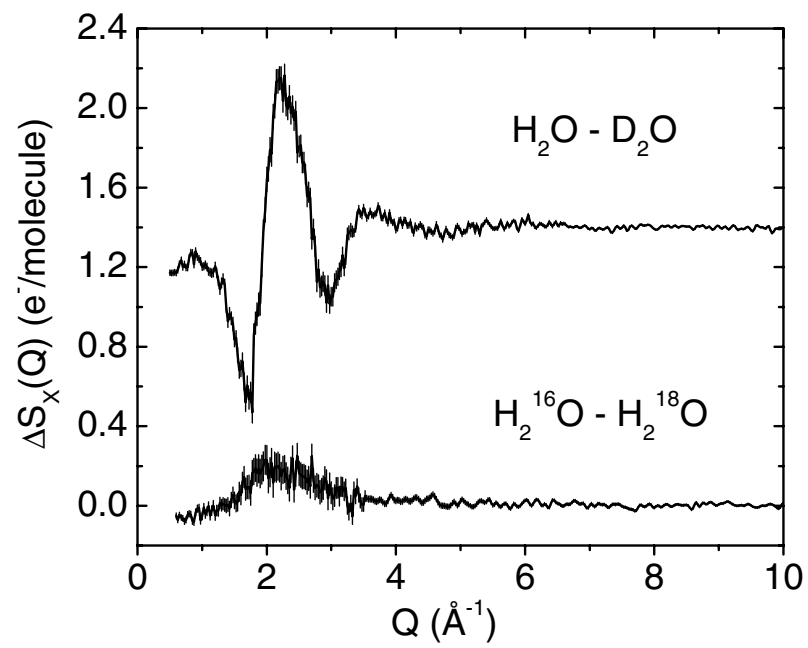

FIG. 1. Difference electronic structure factor, $\Delta S_{X}(Q)$, for light minus heavy water measured at SPring-8. Top: $\mathrm{H}_{2} \mathrm{O}-\mathrm{D}_{2} \mathrm{O}$ at $26.2{ }^{\circ} \mathrm{C}$ (shifted by +1.4 ). Bottom: $\mathrm{H}_{2}{ }^{16} \mathrm{O}-\mathrm{H}_{2}{ }^{18} \mathrm{O}$ at $26.4{ }^{\circ} \mathrm{C}$. Error bars for either data set are shown as vertical lines through the data points. difference in Compton scattering between the two samples, which is negligible for $\mathrm{H}_{2} \mathrm{O}$ and $\mathrm{D}_{2} \mathrm{O}$ [13]. The effect of substitution at the proton sites ( $\mathrm{D}$ for $\mathrm{H}$ ) is dominated by librational effects in the molecule, while ${ }^{16} \mathrm{O}$ versus ${ }^{18} \mathrm{O}$ substitution also has significant contributions from hindered translational and rotational motions [3].

The difference structure factors $\Delta S_{X}^{\mathrm{H}_{2} \mathrm{O}-\mathrm{D}_{2} \mathrm{O}}(Q)$ at $26.2{ }^{\circ} \mathrm{C}$ and $\Delta S_{X}^{\mathrm{H}_{2}{ }^{16} \mathrm{O}-\mathrm{H}_{2}{ }^{18} \mathrm{O}}(Q)$ at $26.4{ }^{\circ} \mathrm{C}$ are shown in Fig. 1. The shape and relative magnitude of $\Delta S_{X}^{\mathrm{H}_{2} \mathrm{O}-\mathrm{D}_{2} \mathrm{O}}(Q)$ is in close agreement with previous studies by Tomberli et al. [1] and Badyal et al. [2]. A study by Bosio et al. [14] has shown that isochoric temperature differentials in water, taken about the density maximum and measured over the range -11 to $40{ }^{\circ} \mathrm{C}$, collapse onto a single curve when divided by the appropriate temperature difference. The isotopic difference structure factors $\Delta S_{X}^{\mathrm{H}_{2} \mathrm{O}-\mathrm{D}_{2} \mathrm{O}}(Q)$ at temperatures of $6,15,23,35$, and $45^{\circ} \mathrm{C}$ are shown in Fig. 2(a). The maximal percent changes in the
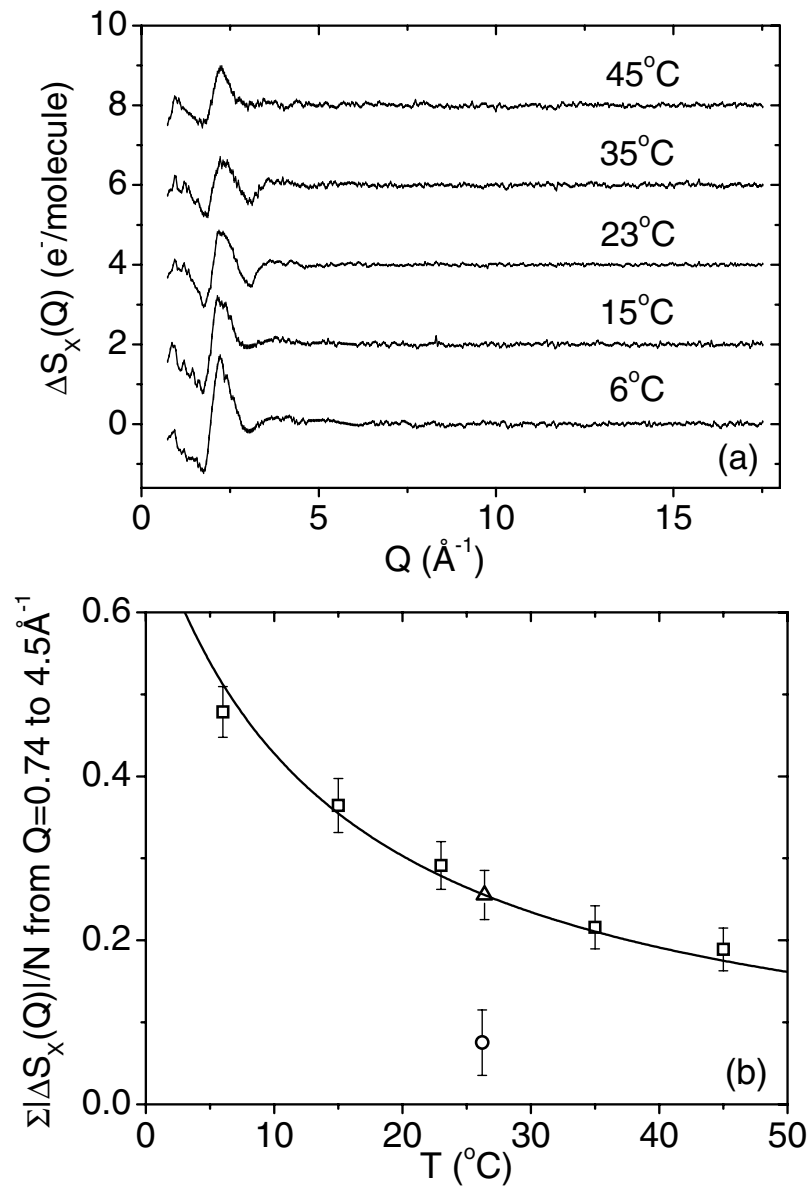

FIG. 2. Isotope quantum effect as a function of temperature measured at HASYLAB. (a) $\Delta S_{X}(Q)$ for $\mathrm{H}_{2} \mathrm{O}-\mathrm{D}_{2} \mathrm{O}$ at (from top to bottom) $45,35,23,15$, and $6{ }^{\circ} \mathrm{C}$ with error bars. (b) Summations over $\Delta S_{X}(Q)$ for $\mathrm{H}_{2} \mathrm{O}-\mathrm{D}_{2} \mathrm{O}$ (squares, HASYLAB; triangle, SPring-8) and $\mathrm{H}_{2}{ }^{16} \mathrm{O}-\mathrm{H}_{2}{ }^{18} \mathrm{O}$ [circle; see Eq. (2) for description of summation]. 
structure factors are $3.0 \%, 2.6 \%, 1.8 \%, 1.4 \%$, and $1.6 \%$, respectively.

To determine the variation in magnitude of the $\mathrm{H}_{2} \mathrm{O}-\mathrm{D}_{2} \mathrm{O}$ structural isotope quantum effect with temperature, we evaluated the quantity

$$
\left(\sum_{Q=0.74}^{4.5} \sqrt{\Delta S_{X}(Q)^{2}}\right) / N
$$

where $N$ is the number of points in the $\Delta S_{X}(Q)$ data. These sums are shown in Fig. 2(b) [15]. The lower limit is the first measured data point at $Q=0.74 \AA^{-1}$, and the highest point at $Q=4.5 \AA^{-1}$ is where the signal difference essentially damps to zero. The fitted curve follows the empirical equation $\Sigma\left|\Delta S_{X}(Q)\right|=(1.37+0.096 \times T)^{-1}$, where $T$ is in ${ }^{\circ} \mathrm{C}$, showing a variation of $\sim 2.5$ in magnitude between 6 and $45^{\circ} \mathrm{C}$. This functional form gave the best fit of several candidates, and because it does not rely on any physical model, we do not warrant its validity outside of these data points. The magnitude of the $\mathrm{H}_{2}{ }^{16} \mathrm{O}-\mathrm{H}_{2}{ }^{18} \mathrm{O}$ substitution is shown in Fig. 2(b) and is found to be $27 \%$ of the $\mathrm{H}_{2} \mathrm{O}-\mathrm{D}_{2} \mathrm{O}$ effect at $26.2^{\circ} \mathrm{C}$ using the procedure described above.

Figure 3 shows the results of Fourier transformation of the $\Delta S_{X}(Q)$ data into real space $\Delta g(r)$ radial distribution functions [16]. The access to slightly more librational modes by $\mathrm{H}_{2} \mathrm{O}$ than $\mathrm{D}_{2} \mathrm{O}$ allows more molecules into the interstitial region, around $3.5 \AA$, between the first and second O-O shells. The shape and the origin (but not the size) of this structural effect have been accurately predicted by the FHMD simulations [5]. In these simulations the water molecule is treated as rigid, indicating that the observed $\mathrm{H}$ versus $\mathrm{D}$ effect for $r>2.5 \AA$ is dominated

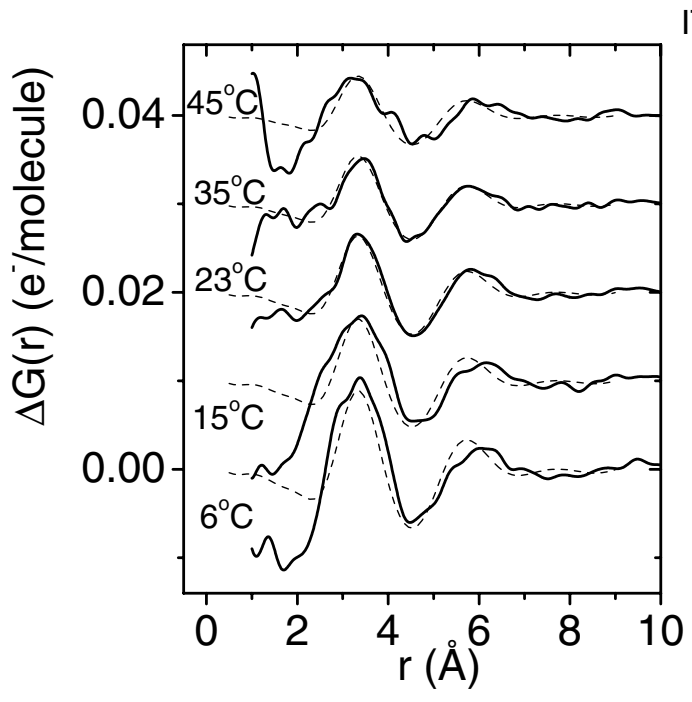

ITD $\left({ }^{\circ} \mathrm{C}\right)$

FIG. 3. Solid lines: Difference correlation functions, $\Delta g(r)$, for $\mathrm{H}_{2} \mathrm{O}-\mathrm{D}_{2} \mathrm{O}$ at (from top to bottom) $45,35,23,15$, and $6{ }^{\circ} \mathrm{C}$. Dashed lines: Isochoric temperature differentials (ITD) from Bosio et al. [14] scaled by $\Delta T$ (from top to bottom): 3.6, 4.3, 5.1, 5.6, and $7.1^{\circ} \mathrm{C}$. by the increased bending about the hydrogen bonds within the intermolecular network [3]. The simulation of Guillot and Guissani [6] has further shown that although water is essentially tetrahedral there is an additional loosely bound fifth molecule (below $3.2 \AA$ ), and as the quantum effects increase, the average distances of the tetrahedra increase, although the relative position of the fifth molecule remains unchanged. The real space $\mathrm{H}_{2} \mathrm{O}-\mathrm{D}_{2} \mathrm{O}$ differences at varying temperatures are also compared to the scaled isochoric temperature derivative (ITD) determined by Bosio et al. [14] in Fig. 3. Structural differences below $2.0 \AA$ are attributed to intramolecular effects in the measured $\mathrm{H}$ versus $D$ data, which are for the most part not present in the ITD. The results show that the $\mathrm{H}$ versus $\mathrm{D}$ isotope effect is comparable to a $3.6^{\circ} \mathrm{C}$ temperature shift in pure $\mathrm{H}_{2} \mathrm{O}$ at $45^{\circ} \mathrm{C}$, which rises to a $7.1^{\circ} \mathrm{C}$ temperature shift at $6^{\circ} \mathrm{C}$.

The $\mathrm{H}_{2}{ }^{16} \mathrm{O}-\mathrm{H}_{2}{ }^{18} \mathrm{O}$ data compared to the difference between the quantized minus classical treatment of center-ofmass modes using path-integral simulations by Kuharski and Rossky [3] are shown in Fig. 4. The experimental data and simulation show approximately similar effects in the region of the nearest neighbor O-O shell at $\sim 2.8 \AA$, as the intensity of the lighter molecule moves to slightly longer distances. However, the experiment shows that the ${ }^{16} \mathrm{O}$ versus ${ }^{18} \mathrm{O}$ substitution effect is small and limited to the first shell, while the simulation predicts large structural rearrangements in both the first and second shells. The results from $\mathrm{H}_{2} \mathrm{O}-\mathrm{D}_{2} \mathrm{O}$ molecular dynamics (MD) simulations of water are also shown [in Fig. 4(b)]. The FHMD simulation has approximately a 3 times larger magnitude even than the largest measured $\mathrm{H}$ versus $\mathrm{D}$ isotope effect at $6{ }^{\circ} \mathrm{C}$, but has excellent agreement with the shape of the effect. In contrast, the CPMD overestimates the abruptness of the $\mathrm{H}_{2} \mathrm{O}-\mathrm{D}_{2} \mathrm{O}$ isotope electron difference in the first $\mathrm{O}-\mathrm{O}$ shell at $2.8 \AA$, but gives a much smaller $\mathrm{H}_{2} \mathrm{O}-\mathrm{D}_{2} \mathrm{O}$ difference curve, which is much closer to the experimental results obtained at $35^{\circ} \mathrm{C}$. A different approach to modeling water using thermodynamically equilibrated ensembles of clusters of molecules qualitatively predicts decreasing quantum difference with increasing temperature, in accord with our results [17].

An important consideration is the effect on H/D substitution in neutron diffraction experiments. A series of neutron experiments may be performed on three samples of different isotopic enrichments to uniquely determine all of the partial correlation functions of water (to a first approximation) [10]. The technique exploits the fact that different isotopes have different neutron scattering cross sections yielding three simultaneous equations. To solve these equations and extract the partial correlation functions, it is assumed that all the different isotopic samples have identical liquid structures. The validity of this technique has been evaluated by Chen et al. in Ref. [7]. Their results show that the structural quantum effects are "likely within the typical uncertainties of NDIS [neutron diffrac- 

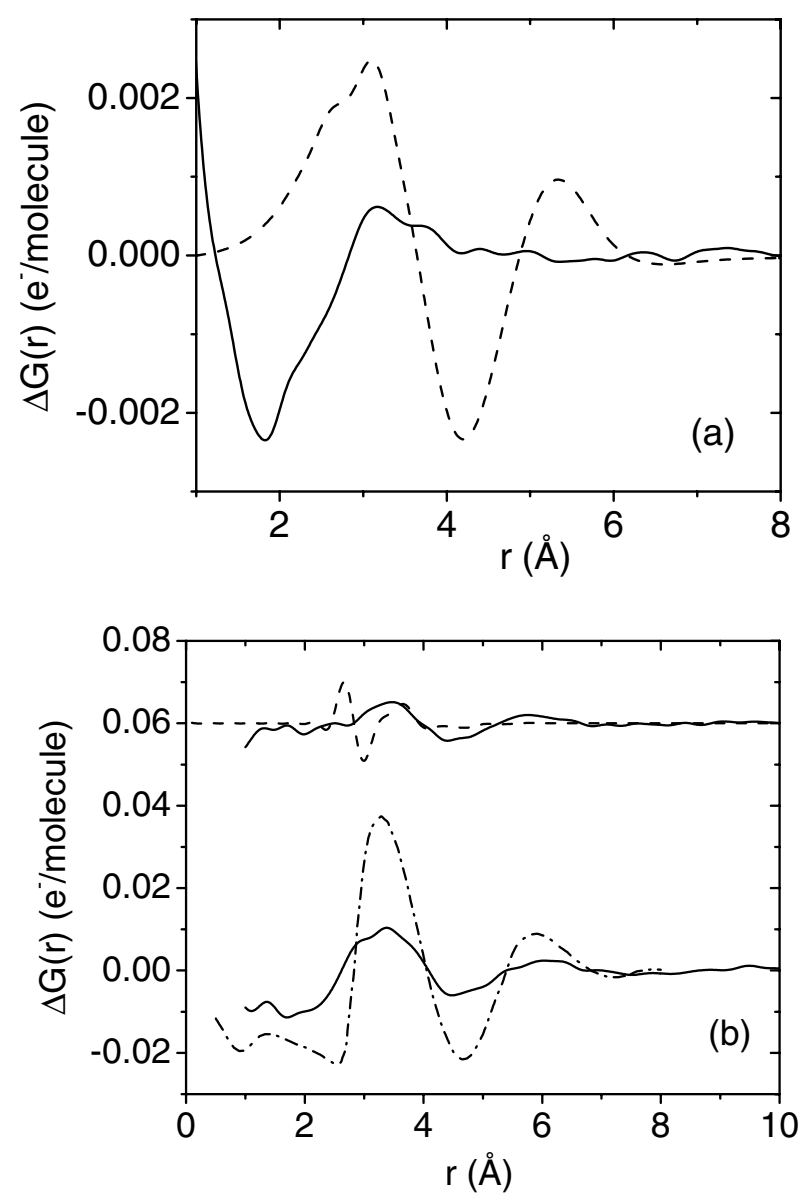

FIG. 4. (a) $\mathrm{H}_{2}{ }^{16} \mathrm{O}-\mathrm{H}_{2}{ }^{18} \mathrm{O}$ difference correlation function (solid line) compared to quantized minus classical path-integral MD simulation from Kuharski and Rossky (dashed line) [3]. (b) A comparison of $\mathrm{H}_{2} \mathrm{O}-\mathrm{D}_{2} \mathrm{O}$ difference data at $35^{\circ} \mathrm{C}$ (upper solid line) with simulation results from Chen et al. [7] (dashed line) and experimental $6{ }^{\circ} \mathrm{C}$ data (lower solid line) with Guillot and Guissani [5] (dot-dashed line). Details are given in Ref. [18].

tion with isotope substitution] experiments at room temperature." The high energy x-ray data presented here show that structural studies making use of the H/D substitution technique in neutron diffraction are significantly minimized at higher temperatures but that they increase with decreasing temperature. Between 6 and $45^{\circ} \mathrm{C}$ the temperature shifts presented in Fig. 3 could be used to constrain the structural difference in the $\mathrm{O}-\mathrm{O}$ partial structure factor measured with NDIS.

We would like to kindly thank Dr. M. Itou for performing additional confirmatory experiments using $176 \mathrm{keV}$. Synchrotron radiation experiments performed at SPring- 8 had approval from the Japan Synchrotron Radiation Research Institute (Proposal No. 2003A0177-ND1-np). Staff at the Hamburger Synchrotronstrahlungslabor HASYLAB are thanked for providing the apparatus and beam time. A part of the work has been performed under the U.S. DOE Contract No. W31-109-ENG-38, and the Canadian participants were supported by a grant from NSERC, Canada.

*Electronic address: benmore@anl.gov

[1] B. Tomberli et al., J. Phys. Condens. Matter 12, 2597 (2000).

[2] Y. S. Badyal et al., J. Chem. Phys. 116, 10833 (2002).

[3] R. A. Kuharski and P. J. Rossky, J. Chem. Phys. 82, 5164 (1985).

[4] G. S. Delbuono, P. J. Rossky, and J. Schnitker, J. Chem. Phys. 95, 3728 (1991).

[5] B. Guillot and Y. Guissani, Fluid Phase Equilib. 150, 19 (1998).

[6] B. Guillot and Y. Guissani, J. Chem. Phys. 108, 10162 (1998).

[7] B. Chen et al., Phys. Rev. Lett. 93, 215503 (2003).

[8] F. Franks, Water, a Comprehensive Treatise (Plenum Press, New York, 1972).

[9] J. R. Grigera, J. Chem. Phys. 114, 8064 (2001).

[10] A. K. Soper, Chem. Phys. 88, 187 (1984).

[11] B. Tomberli et al., J. Phys. Condens. Matter 13, 11405 (2001).

[12] The errors in the difference structure factors were analyzed by varying the normalization factors to minimize the high- $Q$ residual. By this the normalization errors were found to be $<0.1 \%$.

[13] J. H. Root, P. A. Egelstaff, and A. Hime, Chem. Phys. 109, 437 (1986).

[14] L. Bosio, S.-H. Chen, and J. Teixeira, Phys. Rev. A 27, 1468 (1983).

[15] This sum was corrected for the contribution of noise by subtracting the mean of the high- $Q$ noise from each point. The error bars shown in Fig. 2 are the sums of the square roots of the errors on the points from $Q=0.74-4.5 \AA^{-1}$.

[16] Equation (6) from Ref. [1] defines $\Delta g(r)$.

[17] R. Ludwig and F. Weinhold, Phys. Chem. Chem. Phys. 2, 1613 (2000).

[18] To compare to the Car-Parrinello MD simulation (which calculated the electron density difference), we have inverse Fourier transformed the data into $Q$ space and truncated at $Q_{\max }=18 \AA^{-1}$, the limit of our data collection, and transformed the function back to real space. For the Feynmann-Hibbs MD, the atom-atom O-O difference distribution functions were multiplied by $f_{\mathrm{OO}}(Q)^{2}$ in $Q$ space and Fourier transformed back into real space at the $Q_{\max }$ of the data. 